Pediatr. Res. 17: 699 (1983)

\title{
Letter to the Editor: Secretory IgA in Breast Milk
}

\author{
O. G. BROOKE
}

Department of Child Health, St. George's Hospital, London, England

\begin{abstract}
It is sad to read a paper from such distinguished authors which is so poorly reported. The main purpose of the investigation was to show whether breast fed infants of deprived mothers received less IgA than infants of well-off mothers; thus, the critical data were the concentration of IgA in the breast milk and the amount of breast milk received by the infants. On both counts there are problems in the paper as published. There was apparently no standardisation of collection techniques and it is difficult to understand what was done. The milk collected from the Guatemalan mothers was apparently hind milk. This may well not be a representative sample for $\operatorname{IgA}$ concentration. It is not stated how the Ethiopian samples were obtained. Test weighing to estimate volumes of breast milk is notoriously inaccurate. The description of the methods used is again inadequate. It is not clear to the reader whether every feed in $24 \mathrm{~h}$ was measured or who did the
\end{abstract}

measurements, because paramedics were only in the home for 3 h. The interpretation of milk volumes obtained under these conditions must be very cautious.

This leads to another problem. The only clear difference in IgA concentration occurred at 1 month between urban privileged and rural Guatemalans (Table 2). The calculations of daily output of IgA given in Table 3 show a much reduced and no longer significant difference, implying that the rural women had a greater total breast milk output than the urban privileged; however, reference to Table 1 shows that this was apparently not so, the urban privileged having a slightly greater mean milk volume at one month than the rural women. Thus the data are confused and difficult to interpret.

It is a pity that such uncertainties and inconsistencies are allowed past the refereeing stage.

\section{Letter to the Editor: Response}

\author{
JOSE R. CRUZ
}

Instituto de Nutricion de Centro America y Panama, Carretera Roosevelt Zona 11, Guatemala, C.A.

Dr. Brooke is right when he states that, because the purpose of the investigation was to show whether breast fed infants of deprived mothers receive less IgA than infants of well-off mothers, the critical data were the concentration of IgA in the breast milk and the amount of breast milk received by the infants.

In our paper (1) we indicate clearly how the samples were obtained from Guatemalan mothers. Because the methodology was exactly the same in Ethiopia and Sweden we did not consider it necessary to be repetitious in the article.

In regard to the representativeness of the milk sample collected, we had previous information $(2,3)$ that led us to believe that a random sample obtained at any given time during a day is representative of the milk being produced by the mother. Additional information recently obtained in Guatemala, and submitted for publication, indicates that this is exactly the case: the mean IgA content of milk samples taken from 10 mothers in the morning was $0.267 \pm 0.08 \mathrm{~g} /$ liter and the mean concentration for milk samples collected in the afternoon of the same day from the same mothers was $0.263 \pm 0.09$. When we obtained milk specimens from 15 subjects, $5 \mathrm{~min}$ before and 5-10 min after a child's feeding, the concentrations of IgA were $0.270 \pm 0.110$ and 0.270 \pm 0.118 , respectively. We also measured anti-rotavirus $\operatorname{IgA}$ antibodies and did not find any variations in the levels of specific antibodies in the paired samples. All these data indicate that a breast milk sample collected at any given time during the day is representative of the milk the mother produces that given day.
We have found the method to estimate the volume of breast milk ingested by a child in a 24 -h period very accurate. Detecto scales with a sensitivity of $5 \mathrm{~g}$ were used in our study. The details of the standardization of the test weighing are to be published by the World Health Organization in the book Report of a Cross Sectional Study of Quantity and Quality of Breast Milk. The estimated error for intakes of 300,600 , and $900 \mathrm{~g}$ of milk were 6 , 4 , and $3 \%$, respectively and considered acceptable. We described the method in detail in our paper (1); however, the paragraph on page 273 should read: "Paramedical personnel spent a continuous period of $30 \mathrm{~h}$ at each mother's home. The first 6 -h period was aimed at establishing optimal conditions for normal lactation during the observation phase." Obviously, paramedics did the measurements during the continuous 24-h periods.

The last question brought up by Dr. Brooke, regarding differences in $\operatorname{IgA}$ concentration between the urban privileged and rural mothers, but comparable IgA output in $24 \mathrm{~h}$, arises because he examined only the mean values of the volumes and concentrations of IgA. There is great variability in the two parameters in the two population groups, especially among the urban privileged; thus, comparisons of the means is not an adequate analysis. For this reason, we used a non-parametric method, The Wilcoxon test, for the statistical comparisons.

We present the figures, their means and the ranks in Table 1 to allow a better interpretation of the data, as we analyzed them for publication. 